Cultures \& Conflits

64 | hiver 2006

Identifier et surveiller

\title{
Editorial. Identifier et surveiller : les technologies de sécurité
}

Ayse Ceyhan

\section{OpenEdition}

1 Journals

\section{Édition électronique}

URL : http://journals.openedition.org/conflits/2138

DOI : $10.4000 /$ conflits.2138

ISSN : $1777-5345$

Éditeur :

CCLS - Centre d'études sur les conflits lilberté et sécurité, L'Harmattan

\section{Édition imprimée}

Date de publication : 20 décembre 2006

Pagination : 7-9

ISBN : 978-2-296-02667-4

ISSN : 1157-996X

\section{Référence électronique}

Ayse Ceyhan, «Editorial. Identifier et surveiller : les technologies de sécurité », Cultures \& Conflits [En ligne], 64 | hiver 2006, mis en ligne le 06 mars 2007, consulté le 30 mars 2021. URL : http://

journals.openedition.org/conflits/2138; DOI : https://doi.org/10.4000/conflits.2138

Ce document a été généré automatiquement le 30 mars 2021.

Creative Commons License 


\title{
Editorial. Identifier et surveiller : les technologies de sécurité
}

\author{
Ayse Ceyhan
}

1 L'identification et la surveillance - qui étaient a priori conçues comme deux activités distinctes - sont désormais très liées, voire confondues, et cette assimilation est allée croissante avec l'adoption des technologies de sécurité présentées comme un dispositif hautement scientifique de lutte contre les risques et les dangers actuels et futurs. Dans ce cadre, identifier signifie non seulement assigner une identité reconnaissable à un individu par le moyen de critères relativement stables, mais aussi pouvoir connaître ses mouvements, ses actes, ses relations, ses goûts, et même ses projets. Parallèlement, surveiller ne revient plus seulement à suivre la trace d'un individu, mais implique également de pouvoir l'identifier avec certitude à partir de ses habitudes, comportements et itinéraires. Cette confusion est générée par le déploiement de technologies sophistiquées de sécurité qui procèdent à l'identification d'un individu et à sa surveillance à partir de l'examen de ses caractéristiques innées et inchangeables, ce que l'on appelle le « bios».

2 Ce numéro de Cultures \& Conflits a pour objectif de mettre l'accent sur les processus et logiques qui ont mené les Etats, les organismes internationaux - comme l'Organisation de l'aviation civile internationale (OACI) - et les individus à adopter les nouvelles «technologies de sécurité » comme instrument extrêmement puissant de lutte contre les risques et les dangers. Ces technologies sont toutes reliées à des bases de données qui dématérialisent l'identification et la surveillance, les rendant ainsi de plus en plus intrusives. Cela n'est pas sans générer un certain nombre de problèmes quant à la protection de la vie privée et l'encadrement juridique et sans susciter certaines résistances.

3 Les articles de ce numéro prolongent la réflexion entamée dans le cadre de l'étude intitulée « Gendarmerie et technologie : l'impact de la haute technologie sur la sécurité. Analyse comparée » menée pour le compte du Centre de prospective de la Gendarmerie nationale (CPGN) ${ }^{1}$. 
4 L'appellation «technologies de sécurité » est relativement neuve. Bien qu'on la rencontre chez Michel Foucault dans Sécurité, territoire et population ${ }^{2}$, son sens reste plutôt général. Nous l'employons ici comme une expression générique pour désigner les dispositifs d'identification et de surveillance déployés non seulement par les forces de l'ordre mais aussi par le monde de l'entreprise, le monde médical et scientifique ainsi que par les particuliers.

5 L'analyse de ces technologies requiert toutefois deux précautions. La première porte sur la vitesse de l'innovation technologique et la rapidité de sa prolifération. Fondées sur les avancées technologiques récentes, les technologies nouvelles évoluent à une vitesse fulgurante et sont en perpétuelle transformation. De même, à la manière des objets fabriqués par le Démiurge de Platon, ces technologies sont constamment périssables. Elles se caractérisent également par leur ubiquité. Il est donc impossible de les considérer comme des objets immuables et de prétendre les maitriser pour toujours. Notre objectif n'est donc ni de procéder à un état des lieux des nouvelles technologies présentes sur le marché, ni de faire état de leur performance technique.

6 La seconde précaution porte sur la représentation du temps. Les technologies de sécurité manquent d'une échelle de durée pour être justement évaluées. Leur adoption étant un processus en cours, le chercheur ne peut mettre au point une échelle de mesure permettant de comparer les technologies adoptées et d'évaluer leur efficacité et impact en termes relatifs. D'où le problème de manque de recul par rapport à un phénomène non abouti.

7 Compte tenu de ces écueils, nous nous concentrerons sur une réflexion portant sur les contextes et logiques qui conduisent les Etats à transformer la haute technologie en une solution ultime pour faire face aux risques, dangers et incertitudes dans le contexte actuel de sécurisation globale. Ces logiques ne peuvent être examinées indépendamment des enjeux politiques, internationaux, sécuritaires et économiques qui les structurent et les contextes qui les transforment. Pour mieux les saisir, les auteurs ont adopté une posture analytique interdisciplinaire combinant la science politique, la philosophie, la sociologie et le droit.

8 Ayse Ceyhan introduit le numéro en posant un cadre définitionnel qui se démarque des approches essentialistes et déterministes et se propose d'analyser l'adoption des technologies de sécurité en termes de contextes et de dynamiques. Cela permet de montrer combien les transformations contemporaines de la modernité, l'accélération de la globalisation, la privatisation de la sécurité et l'émergence de nouveaux concepts de risque sont génératrices d'incertitudes que les technologies nouvelles sont appelées à maîtriser et à gérer. Mais cet appel n'est pas le seul fait des pouvoirs publics et des entreprises, il jouit également d'une résonance particulière auprès des particuliers du fait de la dynamique de diffusion propre aux technologies nouvelles.

9 L'Etat compose certes avec d'autres acteurs pour adopter les technologies de sécurité dans le cadre d'une gouvernance libérale, mais quel est son véritable enjeu? Dans l'article suivant, Ayse Ceyhan examine le recours aux techniques biométriques comme faisant partie de la problématique de la maîtrise de la fluidité par l'Etat dans le contexte de la globalisation. Défié dans ses fonctions weberiennes, l'Etat cherche à restaurer son monopole sur l'identification des individus et leur surveillance en recourant aux nouvelles technologies. 
10 La biométrie est en passe de devenir un dispositif de surveillance et d'identification dans pratiquement tous les pays de OACI, même en Grande-Bretagne, pays historiquement hostile à l'identification de ses citoyens par des techniques centralisées comme la carte d'identité. Comment les pouvoirs publics justifient-t-ils ce profond revirement? Laurent Laniel et Pierre Piazza examinent les arguments avancés par le gouvernement britannique et particulièrement, celui de la lutte contre le terrorisme.

11 Malgré la dynamique de diffusion qui favorise l'adoption de la biométrie par les particuliers comme une technologie de confort, et les pressions internationales en faveur de son adoption comme technologie de sécurité au niveau global, la biométrie suscite néanmoins des résistances. Ainsi, examinant les différents forums et collectifs qui se sont constitués en France, Pierre Piazza analyse les multiples critiques et contestations suscitées par le projet de la carte INES et montre comment elles ont contribué à retarder son adoption.

12 Les craintes suscitées par les technologies d'identification et de surveillance reposent particulièrement sur leur interconnexion avec des bases de données publiques, privées et transnationales. Sylvia Preuss-Laussinotte analyse les problèmes juridiques posés par ces bases de données qui transforment les données biométriques en véritables données publiques. Elle montre que la constitution de fichiers de sécurité est souvent justifiée au nom d'un «droit à la sécurité », bien souvent confondu avec la notion de « droit à la sûreté ».

13 Quel est le rôle réel de l'Union européenne dans l'adoption des technologies de sécurité ? Sylvia Preuss-Laussinotte affirme que l'Union européenne a choisi d'adopter des technologies de sécurité dans un souci de «cohérence juridique». Toutefois, la réalisation de cet objectif n'est pas de la seule responsabilité de l'Union Européenne, mais également de décisions extérieures : celles des Etats-Unis et de l'OACI.

\section{NOTES}

1.. Cette recherche, dirigée par Ayse Ceyhan et menée avec Jean-Paul Hanon et Sylvia Preuss-Laussinotte, s'est déroulée sur une période de 12 mois, de juillet 2004 à juillet 2005. Ce numéro ne constitue en aucun cas la reproduction du rapport scientifique issu de cette étude.

2.. Foucault reste flou sur cette expression qu'il ne définit pas vraiment. Il évoque l'idée de faire une histoire des technologies de sécurité sans pour autant spécifier ce qu'il entend par cette appellation. Voir Sécurité, territoire, population, Paris, Gallimard / Le Seuil, 2004, pp 9-13 et p. 61. Pour une présentation approfondie de ce que nous entendons par cette expression voir le texte introductif d'Ayse Ceyhan à ce numéro «Technologie et sécurité : une gouvernance libérale dans un contexte d'incertitudes ». 
INDEX

Mots-clés : bases de données, biométrie, surveillance 